\title{
Reduced proximal aortic compliance and elevated wall shear stress after early repair of tetralogy of Fallot
}

\author{
Michal Schäfer, PhD, ${ }^{\text {a,b }}$ Lorna P. Browne, MD, ${ }^{c}$ Gareth J. Morgan, MD, ${ }^{a}$ Alex J. Barker, PhD, ${ }^{c}$ \\ Brian Fonseca, MD, ${ }^{a}$ D. Dunbar Ivy, MD, ${ }^{a}$ and Max B. Mitchell, MD $^{\mathrm{d}}$
}

\section{ABSTRACT}

Objective: Patients with tetralogy of Fallot are at risk for late aortic complications due to progressive aortic root dilation and decreased aortic compliance. Early repair normalizes aortic dimensions by preadolescence. It is not known if early repair normalizes aortic wall histology and compliance or reduces late aortic complications. We used 4-dimensional flow magnetic resonance imaging to determine if children with tetralogy of Fallot repaired in infancy had normal aortic dimensions and to characterize the aortic wall hemodynamic state and luminal flow parameters in these patients.

Methods: Comprehensive aortic analysis with 4-dimensional flow magnetic resonance imaging was performed in 18 patients with tetralogy of Fallot who were repaired in infancy and compared with 18 normal volunteers. Peak systolic and time-averaged wall shear stress, relative area change, and distensibility were evaluated in standardized aortic planes. Qualitative grade scale flow analysis with interactive pathline visualization was used to detect pathologic flow patterns.

Results: Thoracic aortic dimensions did not differ between groups, and all tetralogy of Fallot aortas were in normal range. In the tetralogy of Fallot group, ascending and descending aortic relative area change and distensibility were significantly reduced, and both peak systolic and time-averaged wall shear stress were elevated throughout the aorta. Supra-physiologic systolic helical formations occurred in the ascending aorta of 14 patients with tetralogy of Fallot $(78 \%)$ versus 0 controls.

Conclusions: Despite early repair and normal aortic dimensions, preadolescents and adolescents with tetralogy of Fallot had elevated wall shear stress, increased stiffness, and pathologic systolic flow formations in the proximal aorta. Although early repair normalizes aortic dimensions in childhood, our findings suggest that patients with tetralogy of Fallot remain at risk for late aortic complications. ( $\mathrm{J}$ Thorac Cardiovasc Surg 2018;156:2239-49)

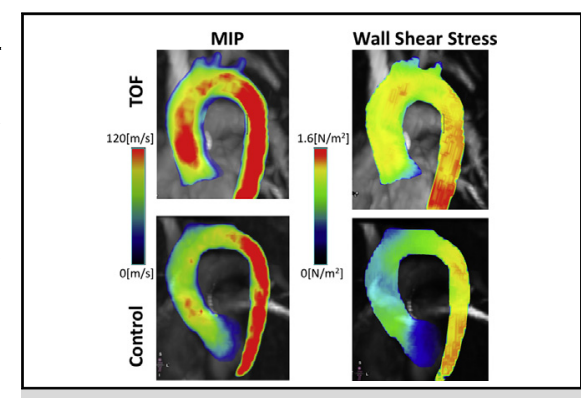

Mean-intensity projection and WSS in the thoracic aorta: elevated in TOF versus control.

\section{Central Message}

Abnormal flow dynamics, elevated WSS, and increased wall stiffness are present in the proximal aorta of young patients with TOF despite early repair and normal aortic size.

\section{Perspective}

Abnormal aortic wall properties may present significant risks to patients with TOF despite early repair. Comprehensive longitudinal 4D flow MRI monitoring of aortic flow patterns, WSS, and elasticity characteristics may benefit these patients by guiding preventative strategies to avoid lethal complications associated with aortic stiffening and dilation.

See Editorial Commentary page 2250

See Editorial page 2237.
Aortopathy is associated with numerous congenital heart diseases, including tetralogy of Fallot (TOF). ${ }^{1-4}$ Significant enlargement of the aortic root and ascending aorta is a near

\footnotetext{
From the ${ }^{\mathrm{a} D i v i s i o n}$ of Cardiology, ${ }^{\mathrm{d}}$ Section of Congenital Heart Surgery, Heart Institute, and 'Department of Radiology, Children's Hospital Colorado, and ${ }^{\mathrm{b}}$ Department of Bioengineering, College of Engineering and Applied Sciences, University of Colorado Denver | Anschutz Medical Campus, Aurora, Colo.

Received for publication June 26, 2018; revisions received Aug 23, 2018; accepted for publication Aug 31, 2018.

Address for reprints: Michal Schäfer, PhD, Department of Cardiology, Children's Hospital Colorado, 13123 E 16th Ave, Aurora, CO 80045-2560 (E-mail: michal. schafer@ucdenver.edu) $0022-5223 / \$ 36.00$

Copyright (C) 2018 Published by Elsevier Inc. on behalf of The American Association for Thoracic Surgery

https://doi.org/10.1016/j.jtcvs.2018.08.081
}

universal finding in neonates with TOF. $^{5}$ Increased proximal aortic size persists in children ${ }^{6}$ and adults ${ }^{7}$ when complete TOF repair is performed beyond the infant stage, and in many cases progressive aortic enlargement ensues. Postrepair aortic enlargement is more common and severe when repair is undertaken in older children and adults. ${ }^{8}$ Previous studies report that $88 \%$ of preadolescent and adolescent

Scanning this QR code will take you to the article title page. 


$\begin{array}{ll}\text { Abbreviations and Acronyms } \\ \text { 4D flow MRI = } & \text { 4-dimensional flow magnetic } \\ & \text { resonance imaging } \\ \text { LV } & =\text { left ventricular } \\ \text { MRI } & =\text { magnetic resonance imaging } \\ \text { RAC } & =\text { relative area change } \\ \text { SSFP } & =\text { steady-state free precession } \\ \text { 3D } & =3 \text {-dimensional } \\ \text { TOF } & =\text { tetralogy of Fallot } \\ \text { 2D } & =\text {-dimensional } \\ \text { WSS } & =\text { wall shear stress } \\ W_{\text {mSS }} & =\text { maximum systolic wall shear stress } \\ \mathrm{WSS}_{\mathrm{TA}} & =\text { time-averaged wall shear stress }\end{array}$

patients with TOF repaired at a mean age of 3.2 years had significant aortic root dilation ( $z$-score $>2$ ), and these patients had increased proximal aortic stiffness compared with controls. $^{6}$ By using noninvasive ${ }^{6,9}$ and catheterization ${ }^{8,10,11}$ methods, other investigators demonstrated increased aortic stiffness in patients with TOF and correlated aortic dilation with increased stiffness. Aortic dilation and elevated wall stiffness are risk factors for life-threatening complications in aortopathies associated with bicuspid aortic valve, coarctation, and Marfan's syndrome. ${ }^{1,12,13}$ Aortic dissection and aortic valve insufficiency can occur with TOF-associated aortopathy. ${ }^{2,14}$ Excess aortic stiffness also increases afterload negatively affecting left ventricular (LV) function, a phenomenon demonstrated even in young children., ${ }^{9,10,13}$

Histopathology in TOF-associated aortopathy closely mirrors Marfan's syndrome and other aortopathies. ${ }^{2,4,15,16}$ Various degrees of medial degeneration are found and have been identified in unrepaired neonates and infants. ${ }^{4,15,16}$ The etiology of TOF-associated aortopathy is speculative. It has been attributed to chronic aortic volume overload, intrinsic genetic-based wall abnormalities, and combinations of these factors. ${ }^{2,4,6,7,10,11,13,15-17}$ Of note, assessments of aortic size progression indicate that proximal aortic size normalizes by the age of 7 years if complete repair is performed during infancy. ${ }^{7,15,18}$ Consequently, the potential to eliminate late complications of TOF-associated aortopathy argues in favor of early repair. It is not known if early repair normalizes aortic histology or wall stiffness, if normalized aortic dimensions are maintained throughout adult life, or if late aortic complications can be avoided.

Four-dimensional flow magnetic resonance imaging (4D flow MRI) augments 2-dimensional (2D) phase-contrast magnetic resonance imaging (MRI) and permits comprehensive characterization of the aortic hemodynamic state to include qualitative and quantitative evaluation of flow formations and comprehensive analysis of wall shear stress (WSS). ${ }^{19}$ We and others have used 4D flow MRI to assess a variety of aortopathies. ${ }^{20-23}$ The purposes of this study were to use 4D flow MRI in preadolescent and adolescent patients with TOF who underwent early complete repair to determine if thoracic aortic dimensions were normal, characterize the flow hemodynamic state of the thoracic aorta, and compare patients with TOF with age-matched controls.

\section{MATERIALS AND METHODS}

As part of a larger retrospective study, we identified preadolescent and adolescent (age $\leq 18$ years) patients with repaired TOF who underwent comprehensive clinically indicated cardiac MRI for the evaluation of right ventricular outflow tract pathology. Patients with bicuspid aortic valve or residual ventricular septal defect were excluded. Eighteen patients who underwent complete repair in infancy ( $\leq 12$ months) formed the TOF group. This age at repair was chosen to assess patients similar to those in prior studies demonstrating aortic size normalization. ${ }^{718}$ Eighteen healthy volunteers of similar age and size with morphologically normal aortas formed the control group. This study was approved by the Colorado Multiple Institutional Review Board as part of larger retrospective study investigating TOF flow hemodynamic changes, and informed consent was obtained for all control subjects.

\section{Cardiac Magnetic Resonance Protocol}

All subjects underwent cardiac magnetic resonance evaluation with a 3T magnet system (Ingenia, Philips Medical Systems, Best, The Netherlands) using a 32-channel coil. Standard 2D cine steady-state free precession (SSFP) long-axis, short-axis, and 4-chamber-axis images were obtained during end-expiratory breath-holds. Additional obliquely oriented 2D cine SSFP planes were acquired along the long axis and short axis of the aortic root and ascending aorta. Aortic size was measured at the sinotubular junction, mid-ascending aorta, aortic arch, isthmus, and proximal descending aorta (Figure 1). Central aortic stiffness was measured in the ascending (between planes S2 and S3) and descending aortas (plane S7) as relative area change (RAC) defined as [(Area $a_{\max }-$ Area $\left._{\min }\right) /$ Area $\left._{\max }\right] \times 100 \%$. Aortic distensibility was approximated as the ratio of RAC and average brachial pulse pressure measured before and immediately after MRI scan. 4D flow MRI was acquired in a sagittal plane covering the mid-thorax with retrospective electrocardiogram gating and a diaphragmatic navigator for assessment of blood flow hemodynamics. Typical scan parameters were as follows: echo times $=2.4$ to $2.6 \mathrm{~ms}$, repetition times $=4.2$ to $5.0 \mathrm{~ms}$, flip angle $\alpha=10^{\circ}$, temporal resolution $=38$ to $48 \mathrm{~ms}$, field of view: 250 to $320 \times 200$ to $250 \mathrm{~mm}^{2}$, 14 cardiac phases, voxel size $=2.0 \times 2.0 \times 2.0$ to $2.8 \mathrm{~mm}^{3}$, VENC $=150 \mathrm{~m} / \mathrm{s}$, acquisition time $=10$ to 12 minutes depending on respiratory gating efficiency.

\section{Wall Shear Stress and Flow Analysis}

4D flow datasets were imported into the CVI42 platform (Version 5.6.6, Circle Cardiovascular Imaging, Calgary, AB, Canada) for qualitative and WSS analyses. Images were corrected for background offset errors and velocity aliasing artifacts. Three-dimensional (3D) direct volume rendering projection then served for 3D segmentation of the thoracic aorta. Hemodynamic WSS represents the tangential force exhibited by a layer of fluid on the endothelial surface and is mathematically defined as a product of the shear rate and shear stress. Shear stress is typically represented by dynamic viscosity (dynamic viscosity is typically considered uniform for all cases). Shear rate is obtained by calculating the velocity derivatives from discrete velocity data obtained by $4 \mathrm{D}$ flow MRI acquisition. Shear hemodynamics were evaluated in 8 standardized planes: S1-S8 (Figure 1). Standard flow hemodynamic indices including maximum velocity $\left(\mathrm{V}_{\max }\right)$, peak systolic flow $\left(\mathrm{Q}_{\mathrm{max}}\right)$, regurgitation fraction, indexed stroke volume, and cardiac index were sampled in each subject from plane S1. 3D segmented aortic 


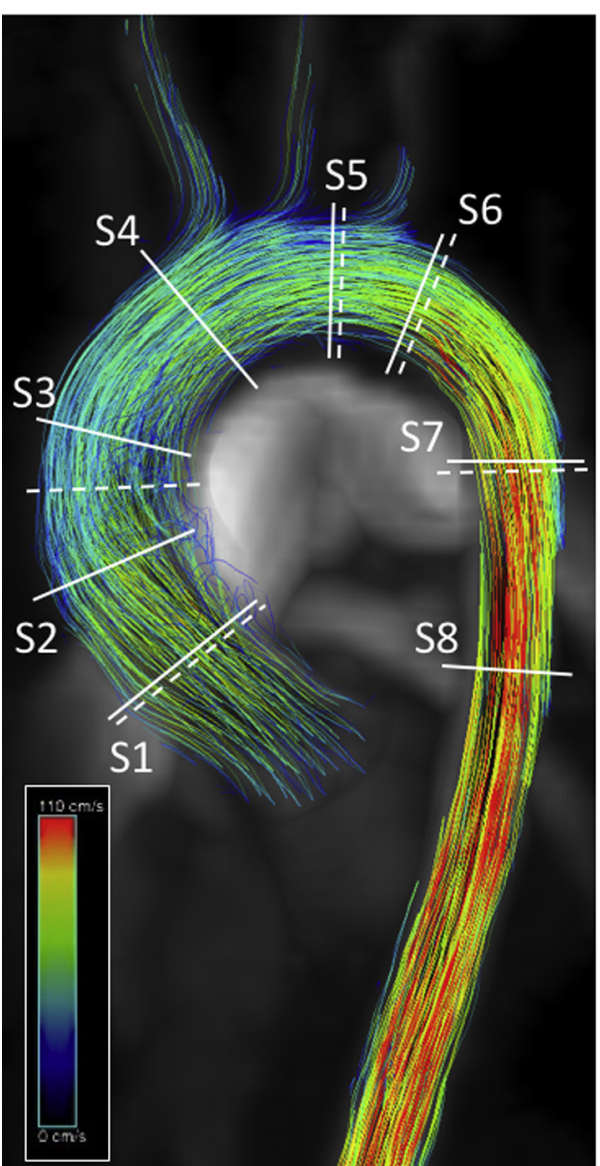

A

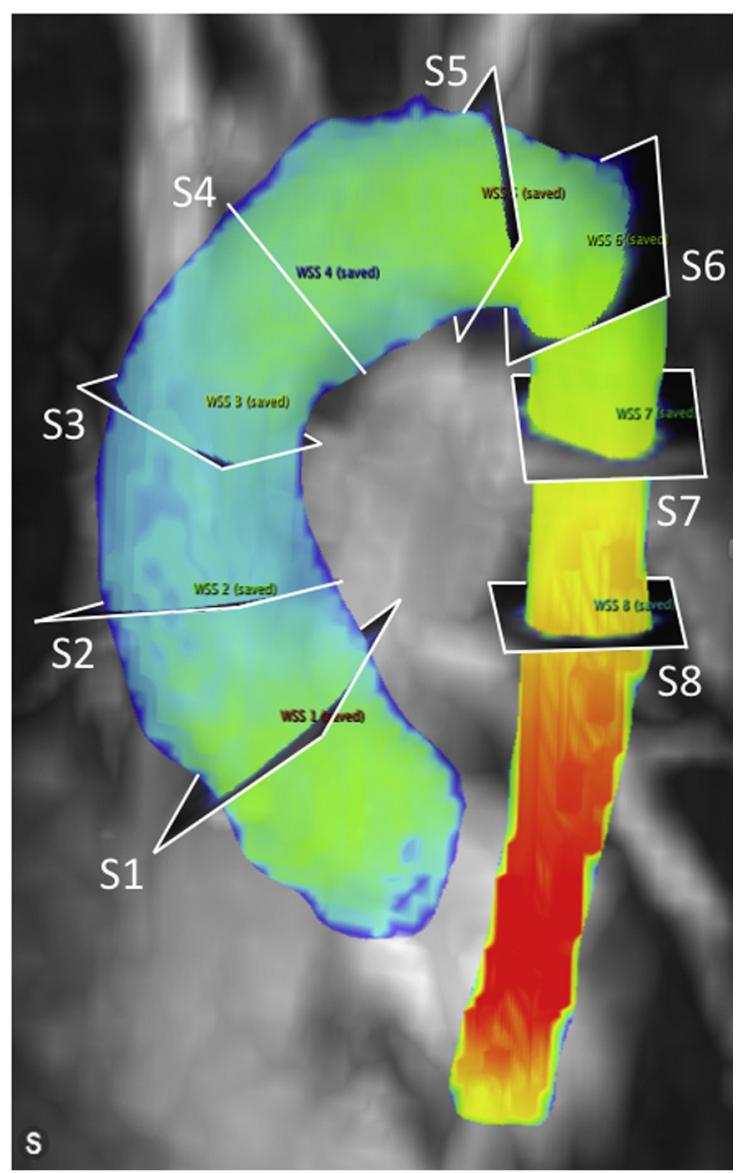

B

FIGURE 1. A, Volumetric pathline representation of thoracic aortic flow in a typical control patient. Standardized evaluation planes for aortic size (dashed lines) and WSS analysis (solid white lines) were selected to comprehensively map shear hemodynamics and geometry from the sinotubular junction through the mid-descending thoracic aorta. Ascending aorta RAC and distensibility measurements were made at the dashed line between planes S2 and S3 and the descending aorta at plane S7. B, 3D heat map of WSS at peak systole with graphical representation of standardized evaluation planes. WSS, Wall shear stress.

contours served for 3D WSS analysis with maximum systolic WSS $\left(\mathrm{WSS}_{\max }\right)$ and time-averaged WSS $\left(\mathrm{WSS}_{\mathrm{TA}}\right)$ recorded from each plane.

Qualitative blood flow analysis was performed throughout systole in the ascending aorta and graded as described by Bürk and colleagues. ${ }^{23}$ This grading scheme uses 2 principle qualitative flow formation shapes-helix and vortex - to define physiologic laminar flow. Grade 0 flow was defined as zero-to-mild vortex/helix formation with rotation less than $180^{\circ}$, Grade 1 flow represented supra-physiologic vortex/helix formation with rotation between $180^{\circ}$ and $360^{\circ}$, and Grade 2 flow was defined as severe vortex/helix formation exceeding $360^{\circ}$ rotation.

\section{Statistical Analysis}

Analyses were performed in JMP (version 13.1 or higher; SAS Institute, Inc, Cary, NC). Variables were checked for the distributional assumption of normality using normal plots, in addition to Kolmogorov-Smirnov and Shapiro-Wilk tests. Demographic and clinical characteristics between groups were compared using Student $t$ test for normally distributed continuous variables. Wilcoxon rank-sum test was used for non-normally distributed variables and chi-square for categoric variables. The relationship between the degree of aortic stiffness and the age at MRI was analyzed by simple linear regression analysis using Pearson R-value. All values are reported as mean \pm standard deviation or median with interquartile ranges.

\section{RESULTS}

Patient characteristics are reported in Table 1. There were no differences in age, body surface area, or sex between groups. The mean age of patients with TOF was 10.3 years (range, 4.1-16.5 years), and the mean age of considered controls was 11.6 (range, 6.2-17.3 years). Twelve patients with TOF had pulmonary stenosis $(67 \%), 4$ patients had pulmonary atresia $(22 \%), 1$ patient had absent pulmonary valve $(6 \%)$, and 1 patient had isolated subpulmonary stenosis $(6 \%)$. Fifteen patients $(83 \%)$ underwent primary complete repair (11 transannular patch, 3 valve sparing, 1 right ventricle-pulmonary artery conduit). Three patients $(16 \%)$ had neonatal systemic-pulmonary shunts followed by shunt takedown and repair with a right ventriclepulmonary conduit. In the TOF group, arch sidedness was 
TABLE 1. Patient characteristics and aortic hemodynamics

\begin{tabular}{|c|c|c|c|}
\hline & $\begin{array}{c}\text { TOF } \\
(n=18)\end{array}$ & $\begin{array}{l}\text { Control } \\
(n=18)\end{array}$ & $P$ value \\
\hline Age (y) & $10.3 \pm 3.2$ & $11.6 \pm 3.4$ & .273 \\
\hline Sex (female) & $6(33 \%)$ & $6(33 \%)$ & .99 \\
\hline $\operatorname{BSA}\left(\mathrm{m}^{2}\right)$ & $1.08 \pm 0.25$ & $1.20 \pm 0.36$ & .123 \\
\hline Interval, repair to MRI (y) & $6.3(4.9-11.9)$ & & \\
\hline \multicolumn{4}{|l|}{ Surgical repair } \\
\hline Transannular patch & $11(61 \%)$ & & \\
\hline Age at repair $(\mathrm{mo})$ & $3.5(2.1-6.2)$ & & \\
\hline Conduit repair & $4(22 \%)$ & & \\
\hline Age at repair $(\mathrm{mo})$ & $9.9(8.8-10.5)$ & & \\
\hline Without patch & $3(17 \%)$ & & \\
\hline Age at repair (mo) & $6.6(5.8-9.3)$ & & \\
\hline Systolic pressure $(\mathrm{mm} \mathrm{Hg})$ & $108 \pm 9$ & $108 \pm 13$ & .944 \\
\hline Diastolic pressure $(\mathrm{mm} \mathrm{Hg})$ & $66 \pm 5$ & $60 \pm 10$ & .088 \\
\hline Pulse pressure $(\mathrm{mm} \mathrm{Hg})$ & $43 \pm 7$ & $48 \pm 7$ & .072 \\
\hline \multicolumn{4}{|l|}{ Thoracic aortic size (mm) } \\
\hline Sinotubular junction & $22 \pm 6$ & $24 \pm 4$ & .219 \\
\hline$z$-score & $1.37 \pm 0.50$ & $1.28 \pm 0.41$ & .322 \\
\hline Ascending aorta & $22 \pm 7$ & $21 \pm 3$ & .471 \\
\hline$z$-score & $1.71 \pm 0.62$ & $1.8 \pm 0.36$ & .591 \\
\hline Arch & $16 \pm 2$ & $18 \pm 3$ & .461 \\
\hline$z$-score & $1.39 \pm 0.52$ & $1.37 \pm 0.21$ & .812 \\
\hline Isthmus & $15 \pm 3$ & $15 \pm 3$ & .649 \\
\hline$z$-score & $1.42 \pm 0.39$ & $1.35 \pm 0.45$ & .419 \\
\hline Descending aorta & $13 \pm 3$ & $14 \pm 1$ & .351 \\
\hline$z$-score & $1.46 \pm 0.41$ & $1.32 \pm 0.15$ & .561 \\
\hline \multicolumn{4}{|l|}{ Aortic hemodynamics } \\
\hline Cardiac index $\left(\mathrm{L} / \mathrm{min} / \mathrm{m}^{2}\right)$ & $3.4 \pm 1.2$ & $3.5 \pm 0.9$ & .487 \\
\hline Stroke volume $\left(\mathrm{mL} / \mathrm{m}^{2}\right)$ & $45 \pm 9$ & $46 \pm 4$ & .898 \\
\hline $\mathrm{V}_{\max }(\mathrm{cm} / \mathrm{s})$ & $108 \pm 21$ & $106 \pm 18$ & .567 \\
\hline Regurgitation fraction (\%) & $0.03(0-1.53)$ & $0.7(0.06-1.97)$ & .663 \\
\hline $\operatorname{Area}_{\max }\left(\mathrm{cm}^{2}\right)$ & $4.4 \pm 1.6$ & $4.3 \pm 1.7$ & .821 \\
\hline $\operatorname{Area}_{\min }\left(\mathrm{cm}^{2}\right)$ & $3.5 \pm 1.4$ & $2.8 \pm 1.2$ & .147 \\
\hline Ascending aortic RAC (\%) & $22 \pm 7$ & $36 \pm 8$ & $<.001 *$ \\
\hline $\begin{array}{l}\text { Ascending aortic } \\
\text { distensibility } \\
(\% / \mathrm{mm} \mathrm{Hg})\end{array}$ & $0.56 \pm 0.23$ & $0.76 \pm 0.23$ & $.005^{*}$ \\
\hline $\begin{array}{l}\text { Descending aortic } \\
\text { RAC }(\%)\end{array}$ & $20 \pm 7$ & $26 \pm 8$ & $.010^{*}$ \\
\hline $\begin{array}{l}\text { Descending aortic } \\
\text { distensibility } \\
(\% / \mathrm{mm} \mathrm{Hg})\end{array}$ & $0.48 \pm 0.10$ & $0.57 \pm 0.16$ & $.012^{*}$ \\
\hline
\end{tabular}

Data are represented as mean \pm standard deviation, or as medians with corresponding interquartile ranges. Thoracic aortic size is measured using luminal diameters. All ascending aortic hemodynamics were measured at $\mathrm{S} 1$ plane. TOF, Tetralogy of Fallot; $B S A$, body surface area; $M R I$, magnetic resonance imaging; $V_{\max }$, maximum velocity; $R A C$, relative area change. ${ }^{*} P<.05$.

left in 10 patients $(56 \%)$ and right in 8 patients (44\%). All controls had a normal left arch. At MRI evaluation, no subjects in either group took antihypertensive medications. There was no difference in blood pressure between groups.

\section{Thoracic Aortic Size and Biomechanics}

Geometric analysis of the thoracic aorta and basic flow hemodynamic comparisons are summarized in Table 1. There were no differences between groups in aortic diameter or respective $z$-scores at any level. There were no differences in $\mathrm{V}_{\max }$, cardiac index, stroke volume index, or aortic regurgitation fraction. Likewise, the 2D cine SSFP-derived maximum and minimum areas measured between levels $\mathrm{S} 2$ and S3 were not different between groups. However, RAC was significantly lower in the TOF group in the ascending aorta $(23 \% \pm 6 \%$ vs $35 \% \pm 8 \%, P<.001)$ and descending aorta $(20 \% \pm 7 \%$ vs $26 \% \pm 8 \%, P=.010)$. The TOF group also had reduced aortic distensibility in the ascending aorta $(0.56 \% \pm 0.16 \% / \mathrm{mm} \mathrm{Hg}$ vs $0.76 \% \pm 0.23 \% / \mathrm{mm}$ $\mathrm{Hg}, P=.005)$ and descending aorta $(0.48 \% \pm 0.10 \% /$ $\mathrm{mm} \mathrm{Hg}$ vs $0.57 \% \pm 0.16 \% / \mathrm{mm} \mathrm{Hg}, P=.012$ ).

Because serial MRI evaluations were not available, each subject's study represents a single snapshot in time. Therefore, we explored the relationship between age at MRI (as a surrogate for time interval since repair in the TOF group) and the degree of aortic stiffness across each cohort using simple linear regression analysis comparing ascending aorta distensibility with age at MRI presented in Figure 2. Aortic distensibility decreased with age in both cohorts (TOF: $\mathrm{R}=-0.63, P=.001$; Control: $\mathrm{R}=-0.54$, $P=.008)$ with very similar slopes. When considering the comprehensive regression across both TOF and control groups, we found mild negative relationship between aortic distensibility and age $(\mathrm{R}=-0.34, P=.043)$.

\section{Wall Shear Stress Analysis}

The plane-specific analyses of $\mathrm{WSS}_{\max }$ and $\mathrm{WSS}_{\mathrm{TA}}$ are summarized in Figure 3. $\mathrm{WSS}_{\max }$ and $\mathrm{WSS}_{\mathrm{TA}}$ metrics were significantly increased in the TOF group at every level compared with control, except the increased $\mathrm{WSS}_{\mathrm{TA}}$ in plane S7 (proximal descending aorta) only approached significance $(P=.067)$. Figure 4 depicts the differences in velocity magnitude and distribution present in the ascending aorta of the typical patient with TOF along with abnormal spatial distribution of $\mathrm{WSS}_{\max }$ between a typical patient with TOF and an age- and size-matched control. WSS $_{\max }$ elevation was most prominent at the outer curve of the sinotubular junction, the branching point of the brachiocephalic artery, and the mid-descending aorta.

\section{Qualitative Flow Analysis}

Pathline visualization in the ascending aorta demonstrated Grade 1 supraphysiologic helix formation present in mid to late systole in 9 patients with TOF (50\%) and Grade 2 large-scale helix formation in 5 patients with TOF $(28 \%)$. Only 4 patients with TOF $(22 \%)$ showed Grade 0 (ie, normal without vortex/helix formations) physiologic flow throughout systole. Grade 0 flow was present in 


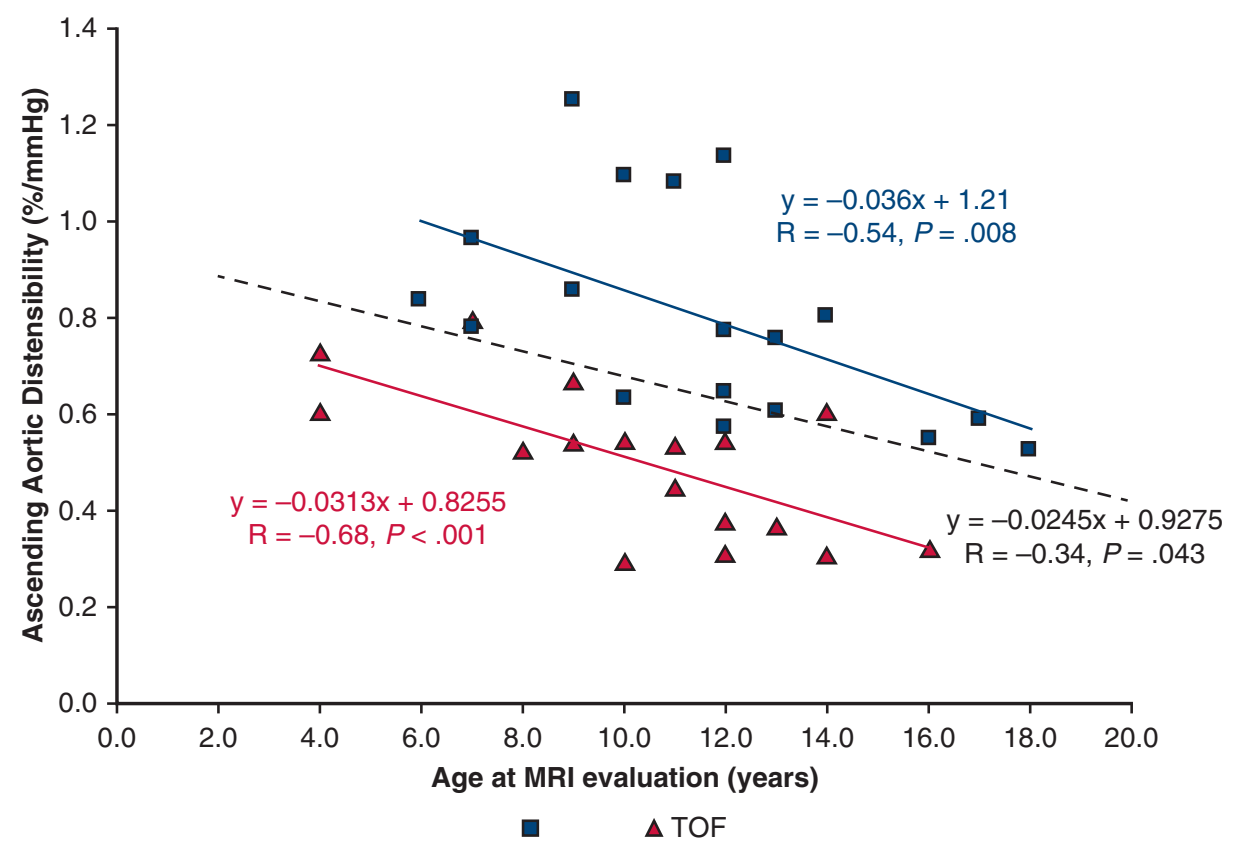

FIGURE 2. Aortic distensibility versus age at MRI assessment. Distensibility correlated negatively with age in both groups. MRI, Magnetic resonance imaging; TOF, tetralogy of Fallot.

all control cases throughout systole. Figure 5 illustrates systolic flow formation in a typical patient with TOF compared with an age- and size-matched control. In both groups, flow remained relatively stable with uniform cohesive pathlines until peak systole (Figure 5, $\mathrm{t}_{2}$ ). In the TOF group, prominent helical formations formed along the medial aspect of the ascending aorta during the late systolic phase (Figure $5, t_{2}-t_{5}$ ). This phenomenon occurred in both right and left aortic arch morphologies (Figure 6).

\section{DISCUSSION}

To our knowledge, this is the first study to assess aortic flow hemodynamics and wall characteristics in patients with TOF at any age and any state of repair using 4D flow MRI. We directed this study at preadolescent and adolescent patients who underwent early TOF repair to characterize the aortic flow hemodynamic state in young patients who underwent surgery consistent with the prevailing standard of care. Our main findings are (1) thoracic aortic dimensions in patients with TOF fell within the normal range and did not differ from healthy control subjects; (2) ascending and proximal descending aortic stiffness was increased (ie, reduced RAC, decreased distensibility) in patients with TOF; (3) WSS was elevated throughout the aorta in the TOF group (S1-S8); (4) supraphysiologic flow formations were prevalent in patients with TOF despite normal aortic size; and (5) there was a near parallel increase in the degree of ascending aortic stiffness with age in patients with TOF and controls, with a pronounced baseline elevated stiffness in patients with TOF.
Right ventricular outflow tract abnormalities constitute the dominant residual anatomic and clinical problems after TOF repair; consequently, follow-up is primarily directed at the right ventricular outflow tract. Proximal aortic enlargement and degenerative histologic changes in the tunica media are characteristic findings in $\mathrm{TOF}^{4,5,24}$ yet relatively little attention is directed at the left ventricle, aortic valve, and aorta until complications of TOF-associated aortopathy ensue. Although rare, aortic dissection has been reported in teens and adults late after TOF repair. ${ }^{14,25}$ Aortic root dilation in patients with TOF results in important aortic valve insufficiency in up to $15 \%$ of patients. ${ }^{2,26}$ From a population vantage point, the most concerning adverse effect of TOF-associated aortopathy results from the obligatory increase in LV afterload that accompanies elevated aortic stiffness. ${ }^{1,9,10}$ Increased afterload results in increased LV mass and LV dysfunction in adults late after TOF repair. ${ }^{9}$ Increased aortic wall stiffness also negatively affects LV ejection, a phenomenon that has been demonstrated in repaired patients with TOF as young as 6 years of age. $^{10}$

Progressive aortic enlargement is more common when complete TOF repair is delayed. ${ }^{2}$ In the current era, repair in infancy is routine. Of note, retrospective and prospective longitudinal studies demonstrate that repair accomplished within the first year of life results in a rapid relative reduction of proximal aortic size over the ensuing 2 years with normalization by age 7 years. ${ }^{7,15,18}$ There are no data that demonstrate if normalized aortic size after early repair is accompanied by histologic remodeling to a more normal 

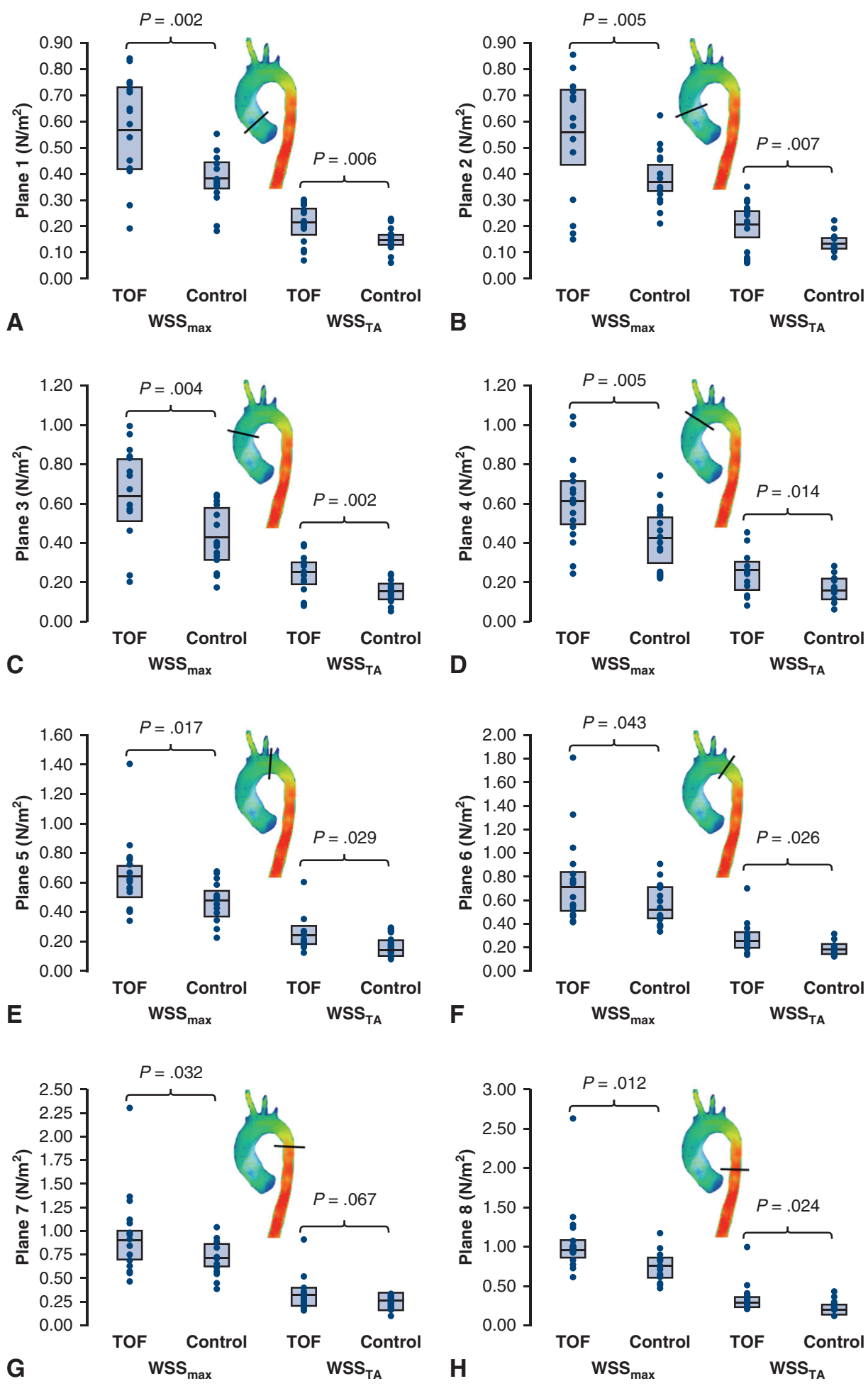

FIGURE 3. Differences in wall shear hemodynamics between the TOF and control groups at specific aortic levels (planes S1-S8; A-H). WSS $\mathrm{max}_{\text {was }}$ significantly elevated in the TOF group at every level (planes S1-S8; A-H). TOF WSS $_{\mathrm{TA}}$ was also significantly increased at every level except plane S7 where elevated $\mathrm{WSS}_{\mathrm{TA}}$ in the TOF group only approached significance. TOF, Tetralogy of Fallot; WSS $_{\max }$, maximum systolic wall shear stress; WSS TA $_{\text {, time- }}$ averaged wall shear stress. 

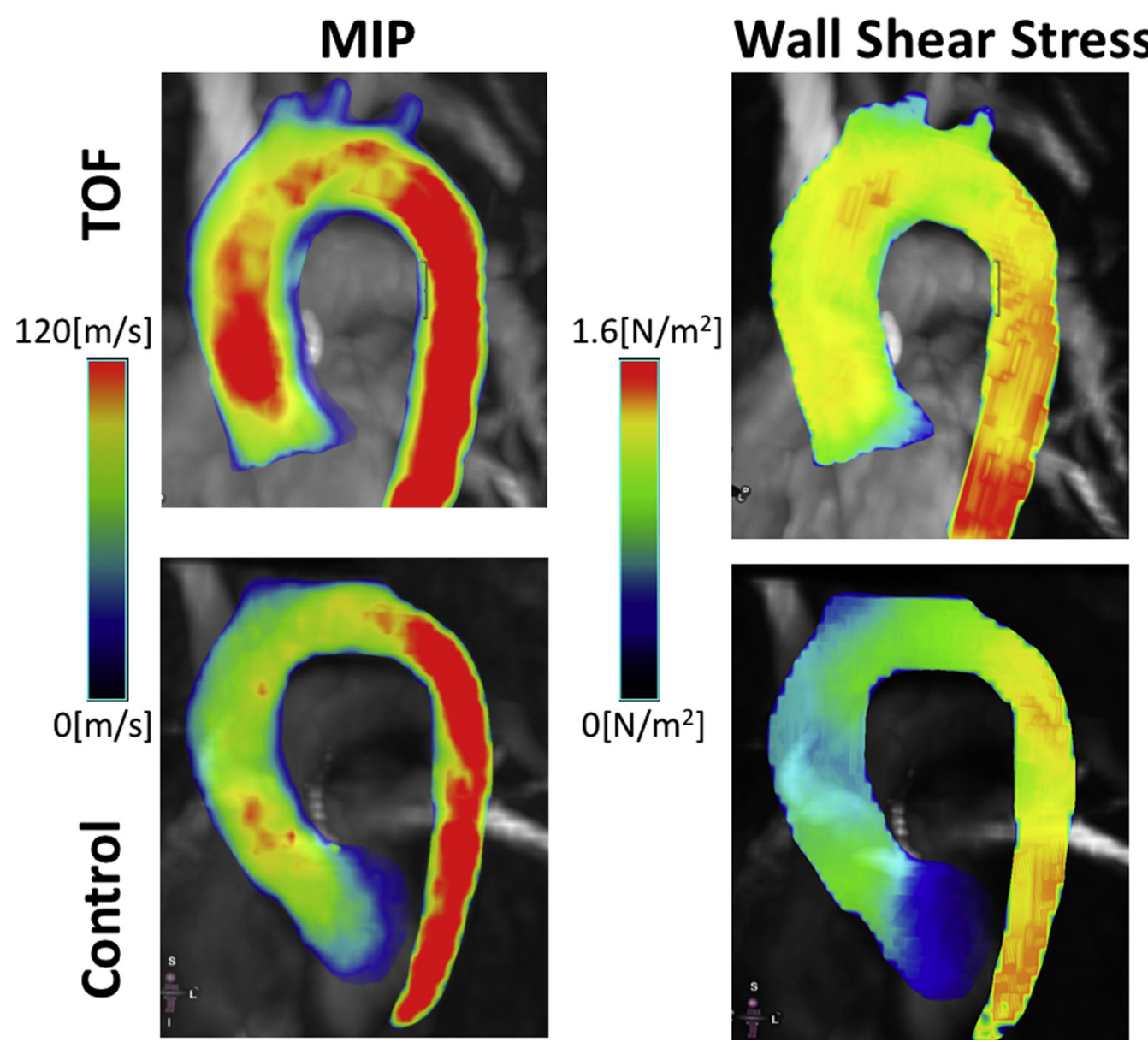

FIGURE 4. Spatial comparison of velocity represented by mean intensity projection and WSS distribution between a typical patient with TOF and typical control. Velocity mean-intensity projection clearly depicts the higher velocity present within the ascending aorta. WSS $\mathrm{max}_{\min }$ the typical patient with TOF was particularly elevated along the outer curve of the ascending aorta and at the branching point of the brachiocephalic artery. MIP, Mean-intensity projection; TOF, tetralogy of Fallot.

state or normalized elasticity, or if normal aortic dimensions are maintained throughout adult life. Furthermore, it is not known if early TOF repair prevents late complications of aortopathy. On the basis of our findings, we conclude that early TOF repair does not normalize aortic wall compliance despite normalized aortic dimensions and that early repair is not likely to prevent late complications from TOF-associated aortopathy.

The exact etiology of TOF-associated aortopathy remains unknown. Explanations include secondary wall damage caused by chronic aortic volume overload, intrinsic genetic predisposition, and combinations of these factors. ${ }^{1,4,12,17}$ Tan and colleagues ${ }^{4}$ described pronounced degenerative changes in the tunica media of the aortic root and ascending aorta in TOF necropsy cases, with ages ranging from neonates to older adults, and concluded that these findings in neonates and young infants support a fundamental intrinsic etiology. Similar findings in aortic cannulation site biopsies and biopsies near the sinotubular junction at complete repair indicate that the magnitude of abnormal changes correlates with age at repair and the degree of aortic dilation. ${ }^{15,16}$ The fact that elimination of excessive aortic volume overload with early repair leads to size normalization suggests that aortic volume overload in utero and early infancy plays a significant role in the pathogenesis of TOF-associated aortopathy.

Aortic dilation in congenital aortopathies including TOF strongly correlates with elevated aortic stiffness. ${ }^{6,11,13,21,27}$ Physiologic measurements of aortic stiffness by both invasive and noninvasive methods consistently demonstrate elevated aortic stiffness in patients with TOF and correlate elevated stiffness with aortic dilation. ${ }^{6,8,10,11}$ Most investigators believe that excessive stiffness precedes and contributes to dilation. ${ }^{6,11,13}$ Seki and colleagues $^{8}$ reported the only data that support this sequence of pathologic progression. They measured 


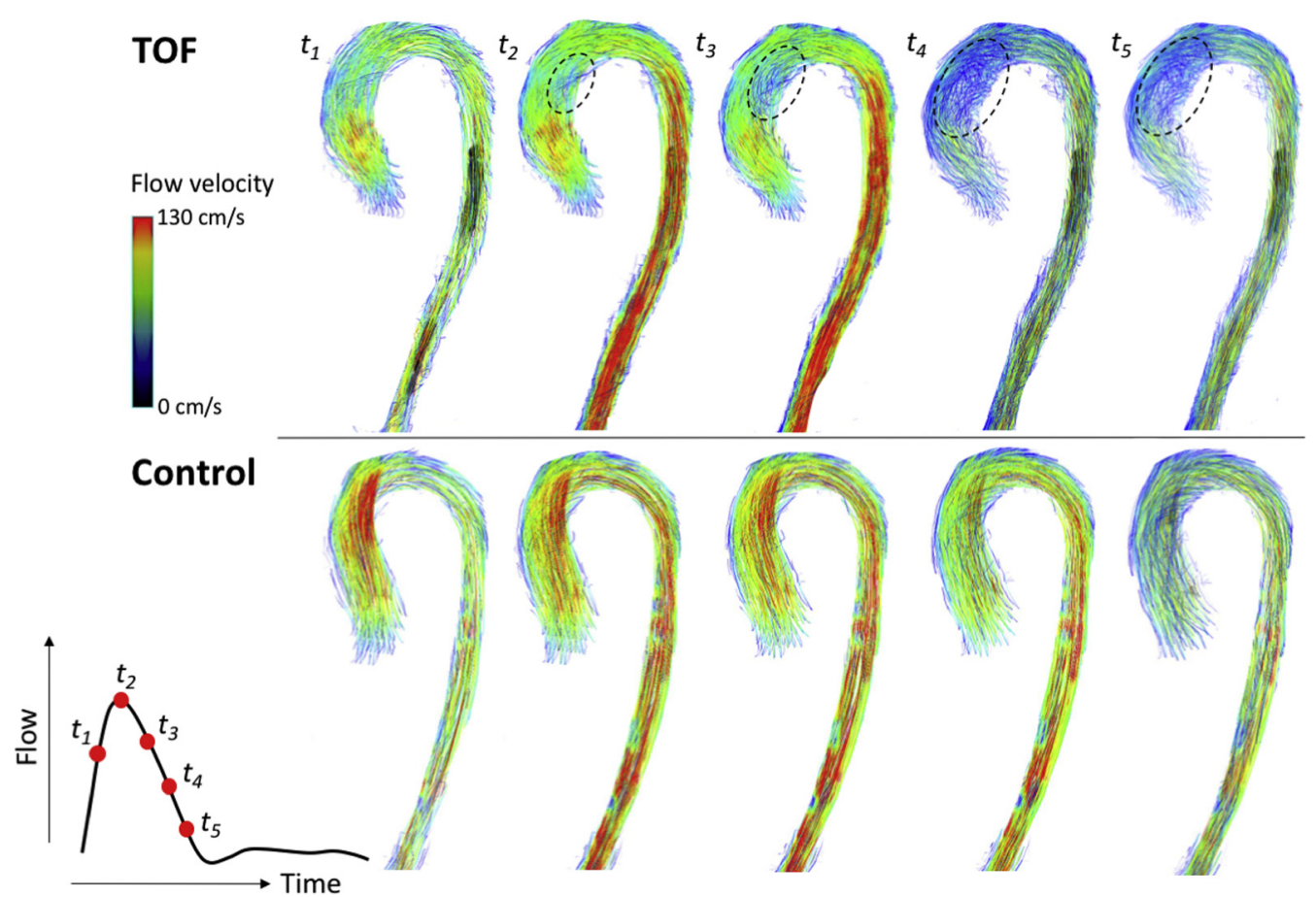

FIGURE 5. Comparison of qualitative flow formations between an age- and size-matched patient with TOF and a control subject represented by pathline visualization throughout systole. Grade 1 and 2 helixes were observed in the ascending aorta of the patient with TOF ( $t_{2}$ to $\left.t_{5}\right)$, whereas flow in the control case remains cohesive throughout systole ( $\mathrm{t}_{1}$ to $\left.\mathrm{t}_{5}\right)$. TOF, Tetralogy of Fallot.

pulse-wave velocity, a measure of aortic stiffness, at preoperative catheterization in 32 patients who underwent complete repair at a mean age of 2 years. They found that the magnitude of baseline aortic stiffness directly correlated with both the rate and the degree of subsequent aortic dilation observed to a mean of 7 years postrepair.

Compared with other methods, 2D phase-contrast MRI assessments of aortic wall properties including size and stiffness are more precise. ${ }^{19,28}$ With appropriate expertise in high-quality image acquisition, 2D phase-contrast and SSFP MRI also allow for specific localized assessment of wall stiffness using flow-area methods or by measuring local RAC or distensibility. ${ }^{27,29}$ Catheterization and echocardiographic methods are limited to more global assessments of thoracic aortic stiffness. With the recent advent of $4 \mathrm{D}$ flow MRI, it is now possible to measure

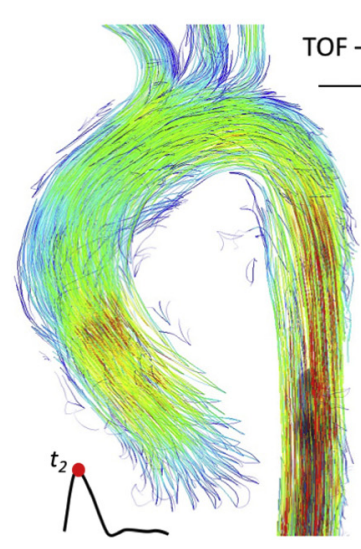

A
TOF - Left Arch

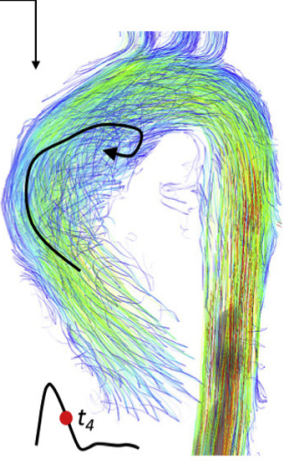

B

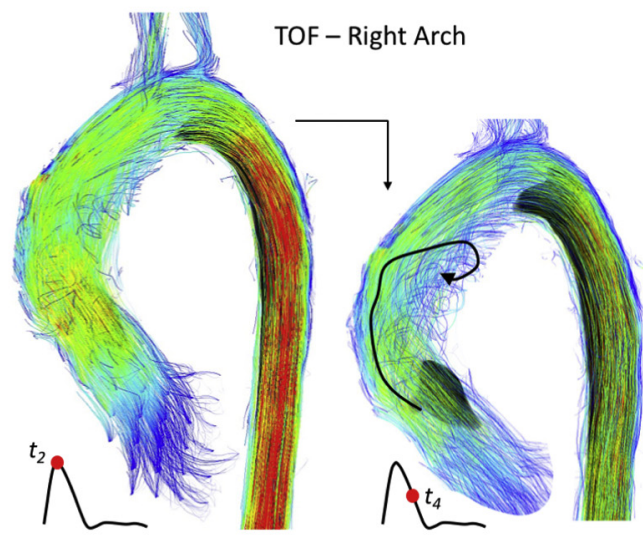

C

D

FIGURE 6. Supra-physiologic helix formations in representative patients with TOF with left and right aortic arches. While flow remained relatively stable until peak systole $-t_{2}$ (A and $C$ ), prominent helical formations were formed along the medial aspect of the ascending aorta during the later systolic $-t_{4}$ phase regardless of the aortic arch morphology (B and D). TOF, Tetralogy of Fallot. 
local WSS in the aorta and to characterize aortic blood flow patterns qualitatively and quantitatively throughout the cardiac cycle. By using advanced MRI methods, we and others have associated abnormal flow hemodynamics and excessive shear forces acting directly on the endothelial surface with corresponding regional histologic findings and abnormal aortic biomechanics in patients with bicuspid aortic valve, aortic stenosis, chronic obstructive pulmonary disease, and connective tissue diseases. ${ }^{20-22,30}$ Observed secondary flow hemodynamic features described in the aforementioned studies in terms of helices and vortices have been closely related to abnormal magnitude and distribution of WSS. Furthermore, abnormal flow shear forces and flow patterns affecting aortic vessel wall remodeling have been associated with histopathologic findings typically found in patients who experienced catastrophic aortic dissections or aneurysmal rupture..$^{21,30}$

Basic-level in vitro studies indicate that increased WSS stimulates mechanotransduction signaling pathways responsible for elastin degradation, medial inflammation, and collagen deposition, thereby augmenting aortic stiffness. $^{31,32}$ In this study, we demonstrated that preadolescent and adolescent patients who underwent complete TOF repair in infancy had significantly elevated WSS in the ascending aorta, transverse arch, and descending aorta. In addition, these patients had elevated aortic stiffness in the ascending and descending aorta, and pathologic flow patterns were common in the ascending aorta despite normal aortic dimensions. We speculate that elevated WSS and nonlaminar flow in the proximal aorta of patients with TOF induces aortic remodeling and the development of aortic stiffness. Baseline 4D flow MRI data on the aortic flow hemodynamic state in unrepaired neonates and young infants would add considerably to our understanding of TOF-related aortopathy; however, MRI studies in this age range require general anesthesia and are not clinically indicated. Further longitudinal studies assessing central aortic stiffness and flow hemodynamics may help to establish a causative role for abnormal shear hemodynamics in the development of aortic root stiffness and dilation in TOF.

Flow hemodynamic shear forces correlate regionally with the composition of aortic media in both in vitro and clinical studies. ${ }^{31,33}$ Significantly elevated shear stress has been linked with local elastin fragmentation, increased concentration of matrix-metalloproteinases, collagen deposition, and overall medionecrosis and fibrosis of vascular wall. ${ }^{31,32}$ Identical intrinsic changes have been described in histologic studies of ascending aortic necropsy specimens in patients with TOF., ${ }^{4,34}$ Of note, the only study assessing descending aortic histology in TOF necropsy cases reported that medial histopathology is limited to the ascending aorta, and the descending aorta is spared. ${ }^{4}$ Previous biomechanical studies support this assertion and report that aortic stiffness in TOF-associated aortopathy is confined to the aortic root and ascending aorta. ${ }^{11,35}$ In contrast, our data demonstrate that the most dramatic changes in WSS and flow formations occur in the ascending aorta and arch; however, we found that WSS and stiffness are also elevated in the descending aorta. This discrepancy is likely due to differences in methodologies used to determine aortic stiffness with a greater sensitivity for advanced MRI. As noted previously, MRI permits highly localized assessment of aortic stiffness, whereas the methods used in previous studies are inherently more global and were likely affected by more normal properties in the distal aorta. Subtle changes in aortic wall composition could lead to aortic stiffness that is detectable by MRI before histologic changes can be readily visualized with light microscopy.

The cause of abnormal flow and WSS in patients with TOF is undetermined. The flow disturbances we observed are likely multifactorial. First, aortic stiffness decreases systolic luminal expansion, thereby elevating WSS and inducing turbulent flow formations. ${ }^{29}$ This mechanism would result in a vicious spiral, leading to the development of aortic dilation. Second, aortic root morphology is distinctively different in TOF with a clockwise rotation of the aortic root due to abnormal conal rotation during embryologic development. ${ }^{34}$ Abnormal geometry of LV outflow may predispose flow toward a nonideal trajectory causing late systolic supraphysiologic helical formations. Interestingly, supraphysiologic flow formations were present in TOF with both left and right aortic arch morphologies. Typical determinants of hemodynamic shear stress-aortic size and stroke volume-were not increased in our TOF cohort compared with controls, suggesting that elevated WSS may be directly associated with reduced RAC, that is, increased aortic stiffness. Additionally, LV-aortic coupling studies in patients with TOF after surgical repair have described elevated aortic pressure wave reflections that negatively affect LV function and flow propagation through the central aorta. ${ }^{9}$ Last, hemodynamic WSS and its associated indices are typically sensitive toward overall vessel wall geometry and inlet conditions in this case defined by LV outflow tract geometry. Consequently, additional parameters affecting the interventricular interactions, such as right ventricular size, septal mechanics, and overall ventricular dyssynchrony, should be considered when assessing the ascending aortic inlet flow conditions. Our future studies will involve LV feature tracking along with quantification of regional flow disturbances inside the LV outflow tract in conjunction with abnormal ascending aortic flow patterns. However, additional studies are required to determine if aortic root rotation and specific arch morphology play a role in the severity of stiffness and flow hemodynamic abnormalities associated with TOF. 
The simultaneous application of computational fluid dynamics would allow for variable alteration of LV outflow tract geometry along with 4D flow MRI datasets providing flow-based inlet conditions. Different types of flow hemodynamic patterns might be then detected and immediately confirmed by 4D flow MRI findings on a patient specific basis.

\section{Study Limitations}

Limitations in this study are inherent to MRI-based pediatric studies and 4D flow MRI imaging. The anesthesia requirement protocol for children aged less than 7 years limited recruitment of controls and mapping of flow hemodynamics in younger patients. In addition, arrhythmias associated with TOF limited effective gating in some studies, resulting in halted 4D flow MRI acquisition. Likewise, the use of healthy children as the control group potentially limits our findings because a prior median sternotomy and cardiac operation could affect the vessel characteristics of the proximal aorta. The small sample size in this study limited exploration of the effect of aortic flow hemodynamics on different types of repair, aortic arch morphologies, and other comprehensive stiffness markers. We did not have adequate clinical data in the TOF group to determine aortic dimensions at the time of early repair. Thus, we assume that early repair led to relative size reduction and normalization of aortic dimensions because the lesions in our TOF cohort would reasonably have been expected to present with increased aortic dimensions. 4D flow MRI assessments in the TOF cohort provided a single snapshot in time. Therefore, it is possible that normalization of aortic size could precede remodeling toward a more normal elastic state and that normalization of aortic wall stiffness in patients who have received early TOF repair could occur with longer follow-up. In the absence of serial MRI evaluations for individual patients with TOF, we compared aortic stiffness with age at MRI as a surrogate for time interval since repair. We found that stiffness increased with age in both patients with TOF and normal children with a substantially elevated baseline in patients with TOF. The consistency of our results across the spectrum of time between early repair and MRI assessment in the TOF cohort argues against the possibility that stiffness may improve over time.

\section{CONCLUSIONS}

This study demonstrated that early complete repair of TOF with normalized aortic dimensions does not normalize aortic wall stiffness. In addition, 4D flow MRI assessment of the aortic hemodynamic state in these patients revealed pathologic findings (increased WSS, supraphysiologic helix formations) that mirror other aortopathies associated with severe aortic complications. Further studies are required to delineate the relationship among elevated aortic stiffness, flow hemodynamics, and aortic morphology in TOF. In addition to standard right heart evaluation, comprehensive longitudinal monitoring of the left heart and flow hemodynamic state in the aorta may be beneficial and may help guide preventative strategies to avoid complications of TOF-associated aortopathy.

\section{Conflict of Interest Statement}

Authors have nothing to disclose with regard to commercial support.

This study was supported by a generous gift from the Rady Family foundation.

\section{References}

1. Zanjani KS, Niwa K. Aortic dilatation and aortopathy in congenital heart diseases. J Cardiol. 2013;61:16-21.

2. Niwa K, Siu SC, Webb GD, Gatzoulis MA. Progressive aortic root dilatation in adults late after repair of tetralogy of Fallot. Circulation. 2002;106:1374-8.

3. Niwa K. Aortic root dilatation in tetralogy of Fallot long-term after repair-histology of the aorta in tetralogy of Fallot: evidence of intrinsic aortopathy. Int J Cardiol. 2005;103:117-9.

4. Tan JL, Davlouros PA, McCarthy KP, Gatzoulis MA, Ho SY. Intrinsic histological abnormalities of aortic root and ascending aorta in tetralogy of Fallot: evidence of causative mechanism for aortic dilatation and aortopathy. Circulation. 2005;112:961-8.

5. Jatavan P, Tongprasert F, Srisupundit K, Luewan S, Traisrisilp K, Tongsong T. Quantitative cardiac assessment in fetal tetralogy of Fallot. $J$ Ultrasound Med. 2016;35:1481-8.

6. Chong WY, Wong WHS, Chiu CSW, Cheung YF. Aortic root dilation and aortic elastic properties in children after repair of tetralogy of Fallot. Am J Cardiol. 2006;97:905-9.

7. Bhat AH, Smith CJ, Hawker RE. Late aortic root dilatation in tetralogy of Fallot may be prevented by early repair in infancy. Pediatr Cardiol. 2004;25:654-9.

8. Seki M, Kurishima C, Saiki H, Masutani S, Arakawa H, Tamura M, et al. Progressive aortic dilation and aortic stiffness in children with repaired tetralogy of Fallot. Heart Vessels. 2014;29:83-7.

9. Shiina Y, Murakami T, Kawamatsu N, Niwa K. Aortopathy in adults with tetralogy of Fallot has a negative impact on the left ventricle. Int J Cardiol. 2017;228: $380-4$.

10. Senzaki H, Iwamoto Y, Ishido H, Matsunaga T, Taketazu M, Kobayashi T, et al. Arterial haemodynamics in patients after repair of tetralogy of Fallot: influence on left ventricular after load and aortic dilatation. Heart. 2008;94:70-4.

11. Seki M, Kurishima C, Kawasaki H, Masutani S, Senzaki H. Aortic stiffness and aortic dilation in infants and children with tetralogy of Fallot before corrective surgery: evidence for intrinsically abnormal aortic mechanical property. Eur J Cardiothorac Surg. 2012;41:277-82.

12. Yetman AT, Graham T. The dilated aorta in patients with congenital cardiac defects. J Am Coll Cardiol. 2009;53:461-7.

13. Niwa K. Aortopathy in congenital heart disease in adults: aortic dilatation with decreased aortic elasticity that impacts negatively on left ventricular function. Korean Circ J. 2013;43:215-20.

14. Konstantinov IE, Fricke TA, D’Udekem Y, Robertson T. Aortic dissection and rupture in adolescents after tetralogy of Fallot repair. J Thorac Cardiovasc Surg. 2010;140:e71-3.

15. François K, Creytens D, De Groote K, Panzer J, Vandekerckhove K, De Wolf D, et al. Analysis of the aortic root in patients with tetralogy of Fallot undergoing early repair: form follows function. J Thorac Cardiovasc Surg. 2014;148:1555-9.

16. Chowdhury UK, Mishra AK, Ray R, Kalaivani M, Reddy SM, Venugopal P. Histopathologic changes in ascending aorta and risk factors related to histopathologic conditions and aortic dilatation in patients with tetralogy of Fallot. J Thorac Cardiovasc Surg. 2008;135:69-77. 77.e1-11.

17. Seki M, Kuwata S, Kurishima C, Nakagawa R, Inuzuka R, Sugimoto M, et al. Mechanism of aortic root dilation and cardiovascular function in tetralogy of Fallot. Pediatr Int. 2016;58:323-30.

18. François K, Zaqout M, Bové T, Vanderkerckove D, De Groote K, Panzer J, et al. The fate of the aortic root after early repair of tetralogy of Fallot. Eur J Cardiothorac Surg. 2010;37:1254-8. 
19. Dyverfeldt P, Bissell M, Barker AJ, Bolger AF, Carlhäll CJ, Ebbers T, et al. 4D flow cardiovascular magnetic resonance consensus statement. J Cardiovasc Magn Reson. 2015; 17:72.

20. Schäfer M, Kheyfets VO, Barker AJ, Stenmark K, Hunter KS, McClatchey PM, et al. Reduced shear stress and associated aortic deformation in the thoracic aorta of patients with chronic obstructive pulmonary disease. J Vasc Surg. 2018;68: 246-53.

21. Barker AJ, Markl M, Bürk J, Lorenz R, Bock J, Bauer S, et al. Bicuspid aortic valve is associated with altered wall shear stress in the ascending aorta. Circ Cardiovasc Imaging. 2012;5:457-66.

22. van der Palen RLF, Barker AJ, Bollache E, Garcia J, Rose MJ. Altered aortic 3D hemodynamics and geometry in pediatric Marfan syndrome patients. J Cardiovasc Magn Reson. 2017;19:30.

23. Bürk J, Blanke P, Stankovic Z, Barker AJ, Russe M, Murtagh G, et al. Evaluation of $3 \mathrm{D}$ blood flow patterns and wall shear stress in the normal and dilated thoracic aorta using flow-sensitive 4D CMR. J Cardiovasc Magn Reson. 2012;14:84.

24. Wu LH, Wang N, Xie HN, Du L, Peng R. Cardiovascular z-scores in fetuses with tetralogy of Fallot. Ultrasound Obstet Gynecol. 2014;44:674-81.

25. Frischhertz BP, Shamszad P, Pedroza C, Milewicz DM, Morris SA. Thoracic aortic dissection and rupture in conotruncal cardiac defects: a populationbased study. Int J Cardiol. 2015;184:521-7.

26. Dodds GA III, Warnes CA, Danielson GK. Aortic valve replacement after repair of pulmonary atresia and ventricular septal defect or tetralogy of Fallot. J Thorac Cardiovasc Surg. 1997;113:736-41.

27. Schafer M, Browne LP, Truong U, Jaggers J, Mitchell MB, Malone L, et al. Aortic stiffness in adolescent Turner and Marfan syndrome patients. Eur J Cardiothorac Surg. 2018;0:1-7.
28. Nayak KS, Nielsen J-F, Bernstein MA, Markl M, Gatehouse PD, Botnar RM, et al. Cardiovascular magnetic resonance phase contrast imaging. J Cardiovasc Magn Reson. 2015;17:71.

29. Schäfer M, Ivy DD, Abman SH, Barker AJ, Browne LP, Fonseca B, et al Apparent aortic stiffness in children with pulmonary arterial hypertension. Circ Cardiovasc Imaging. 2017;10.

30. Guzzardi DG, Barker AJ, van Ooij P, Malaisrie SC, Puthumana JJ. Valve-related hemodynamics mediate human bicuspid aortopathy. J Am Coll Cardiol. 2015;66: 892-900.

31. Davies PF. Hemodynamic shear stress and the endothelium in cardiovascular pathophysiology. Nat Clin Pract Cardiovasc Med. 2009;6:16-26.

32. Tzima E, Irani-Tehrani M, Kiosses WB, DeJana E, Schultz DA, Engelhardt B, et al. A mechanosensory complex that mediates the endothelial cell response to fluid shear stress. Nature. 2005;437:426-31.

33. Garcia J, Barker AJ, Murphy I, Jarvis K, Schnell S, Collins JD, et al. Four-dimensional flow magnetic resonance imaging-based characterization of aortic morphometry and haemodynamics: impact of age, aortic diameter, and valve morphology. Eur Heart J Cardiovasc Imaging. 2016;17:877-84.

34. Isaaz K, Cloez J, Marcon F, Worms A, Pernot C. Pathophysiology and natural history. Is the aorta truly dextroposed in tetralogy of Fallot? Circulation. 1986;5: 892-9.

35. Saiki H, Kojima T, Seki M, Masutani S, Senzaki H. Marked disparity in mechanical wall properties between ascending and descending aorta in patients with tetralogy of Fallot. Eur J Cardiothorac Surg. 2012;41:570-3.

Key Words: tetralogy of Fallot, aorta, flow hemodynamics, MRI 